\title{
Application of Nanocarbons Modified with Anthraquinone Polycondensation Polymer to Electrochemical Double Layer Capacitance
}

\author{
K. Nishiyama ${ }^{\mathrm{a}}$, K. Yamaguchi ${ }^{\mathrm{b}}, \mathrm{H}$. Minamoto ${ }^{\mathrm{c}}, \mathrm{S}$. Yoshimoto ${ }^{\mathrm{d}}$, and M. Takafuji ${ }^{\mathrm{a}}$ \\ a Faculty of Advanced Science and Technology, Kumamoto University, \\ 2-39-1 Kurokami, Chuo-ku, Kumamoto, 860-8555, Japan \\ b Department of Material Science and Applied Chemistry, Kumamoto University, 2-39-1 \\ Kurokami, Chuo-ku, Kumamoto, 860-8555, Japan \\ ${ }^{c}$ Graduate School of Science and Technology, Kumamoto University, \\ 2-39-1 Kurokami, Chuo-ku, Kumamoto, 860-8555, Japan \\ ${ }^{d}$ Institute of Industrial Nanomaterial, Kumamoto University, \\ 2-39-1 Kurokami, Chuo-ku, Kumamoto, 860-8555, Japan
}

By one-pot polymerization of anthraquinone derivative having two hydroxy groups and aliphatic amine, four kinds of carbon blacks were successfully modified. Although the modified CBs were unstable in the alkaline media, the electrodes show stable cyclic voltammograms in neutral and acidic media. All the modified CBs shows the function as the electrochemical double layer capacitance. Especially, the largest capacitance was obtained in $3 \mathrm{M} \mathrm{H}_{2} \mathrm{SO}_{4}$.

\section{Introduction}

Carbon-based fine particles have been widely used as fillers for enhancing the reinforcement for bulk materials $(1,2)$. In addition, the excellent physical stability and unique chemical properties of these fine particles expand their potential utility in electroconductive materials $(3,4)$, carriers for catalysis $(5,6)$, packing materials such as pigment and colorant for inks, paints, and resins. Carbon black is one of the nanocarbons and have been widely used as black materials such as pigment and colorant for inks, paint

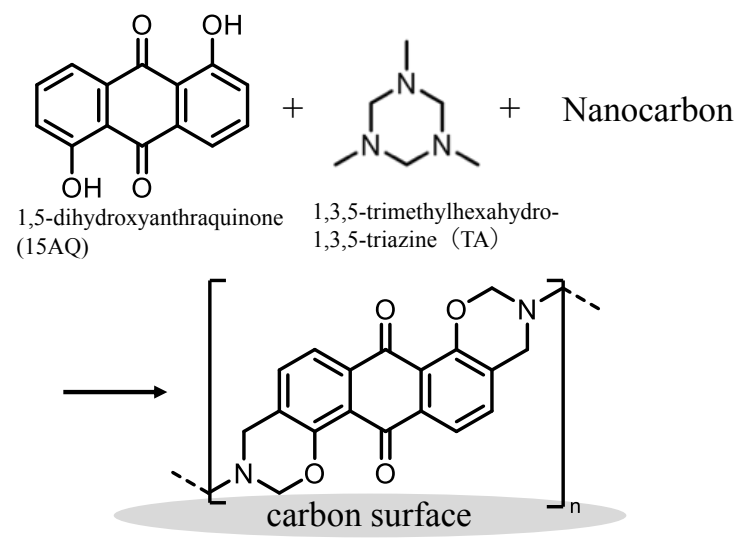

Figure 1. Chemical structure s of (a) 1,5-dihydroxyanthraquinone (15AQ) and (b) 1,3,5trimethyl-1,3,5-triazinane (TA). (c) Estimated structure of polycondensation polymer on nanocarbon surface.

Recently, we have reported that one-pot polymerization of 1.5dihydoroxynaphtalene (DHN) and 1,3,5-trimethylhexahydro-1,3,5-triazinane(TA) in ethanol or other related solvents without any additives, and subsequent calcination at 
relatively enables us to control the diameter of the resultant particle in the range of submicron to a couple of microns (7). In addition, the organic fluorescent nanogel particles showing solvatochromism by the one-pot polymerization of aromatic compounds having dihydroxy group and TA have been reported (8). Furthermore, this one-pot polymerization method enables us to modify the silica nanoparticles $(7,8)$.

On the other hand, $\mathrm{CB}(9,10)$ and other carbon material such as activated carbon $(11)$ and glassy carbon (12) have been studied as a material for the electrochemical double layer capacitance (EDLC) for their superior properties. Also, the electroconductive polymer including redox polymer have been paid attention to the application to the pseudo redox capacitor device (13-15).

In this study we report that the nanocarbons modified with an anthraquinone polycondensation polymer prepared by this one-pot polymerization method using an aromatic hydrocarbon with two $\mathrm{OH}$ groups and TA function as the EDLC.

\section{Experimental}

1,5-Dihydroxyanthraquinone (15AQ) and 1,3,5-trimethyl-1,3,5-triazinane (TA) were purchased from Tokyo Chemical Industry Co., Ltd. and used as received. Four types of carbon black (CB) powders (DENKA Black 2137, 2175, FX-35, and HS-100) were gifted from DENKA Co. Ltd, Japan and were abbreviated as 2137, 2175, FX-35, and HS-100, respectively. Mineral oil was purchased from BAS Ltd. Milli-Q water was used for the preparation of all the solutions. Carbon paste electrode (CPE) and mineral oil were purchased from BAS Ltd. Other chemical reagents were G.R. grade and used as received.

The typical preparation procedure of modified $\mathrm{CB}$ is as follows: $15 \mathrm{mM}$ each of 15AQ and TA was mixed and dissolved in cyclohexanone at room temperature. After 20 $\mathrm{mg}$ of $\mathrm{CB}$ was added to the solution, it was heated at $90{ }^{\circ} \mathrm{C}$ under gentle stirring for $6 \mathrm{~h}$. The modified CBs were collected by centrifugation (10000 rpm, $30 \mathrm{~min})$. The precipitated products, modified $\mathrm{CBs}$ were washed with cyclohexanone and dried in vacuo. Cyclic voltammetry $(\mathrm{CV})$ and potential step chronocoulometry $(\mathrm{CC})$ were performed with an ALS model 621 electrochemical analyzer. A platinum plate and $\mathrm{Ag} / \mathrm{AgCl}(\mathrm{KCl}$ sat.) were used as a counter and reference electrode, respectively. $\mathrm{CB}$ electrodes were prepared by mixing $\mathrm{CB}$ powder with mineral oil. The mixing ratio of $\mathrm{CB}$ powder with the mineral oil was changed from $50 \%$ to $95 \%$. The mixture was introduced into a CPE with $6 \mathrm{~mm}$ diameter. All the capacitances were calculated according to the method using CVs (16).

The morphology of the CBs was observed by scanning electron microscopy (SEM) using a SU8000 microscope (Hitachi High-Technologies, Japan) operating at 5.0 KV. UVvis spectra were recorded on a Shimadzu UV-3100 PC spectrophotometer.

\section{Results and Discussion}




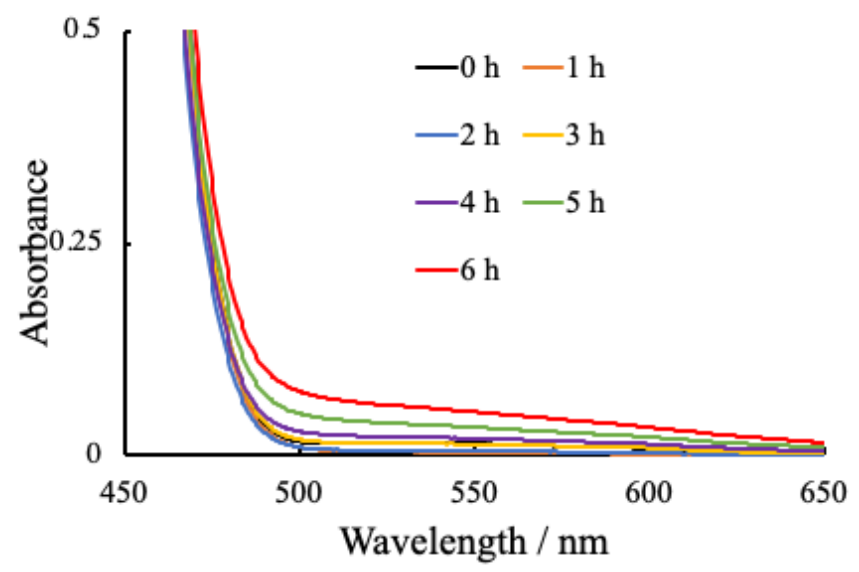

Figure 2. Change in visible spectra during the reaction of $15 \mathrm{mM} 15 \mathrm{AQ}$ and $15 \mathrm{mM} \mathrm{TA}$ in cyclohexanone. Reaction tempararure: $90^{\circ} \mathrm{C}$. The visible spectra before heating $(0 \mathrm{~h})$, after heating for $1,2,3,4,5$, and 6 hours were shown.

Figure 2 shows change in visible spectra during the reaction of $15 \mathrm{mM} 15 \mathrm{AQ}$ and $15 \mathrm{mM}$ TA in cyclohexanone at $90^{\circ} \mathrm{C}$. Here, no CBs were added in the solution to observe the reaction of $15 \mathrm{AQ}$ and TA in solution. Before heating (reaction time: $0 \mathrm{~h}$ ), no absorption band from $500 \mathrm{~nm}$ to $650 \mathrm{~nm}$ were observed. New absorption bands between $500 \mathrm{~nm}$ and $650 \mathrm{~nm}$ were observed as the reaction time increased. The appearance of new absorption band in the relative long wavelength region would indicate that the reaction between $15 \mathrm{AQ}$ and TA proceeded in solution. No further change in the spectrum was observed after heating for more than 6 hours.
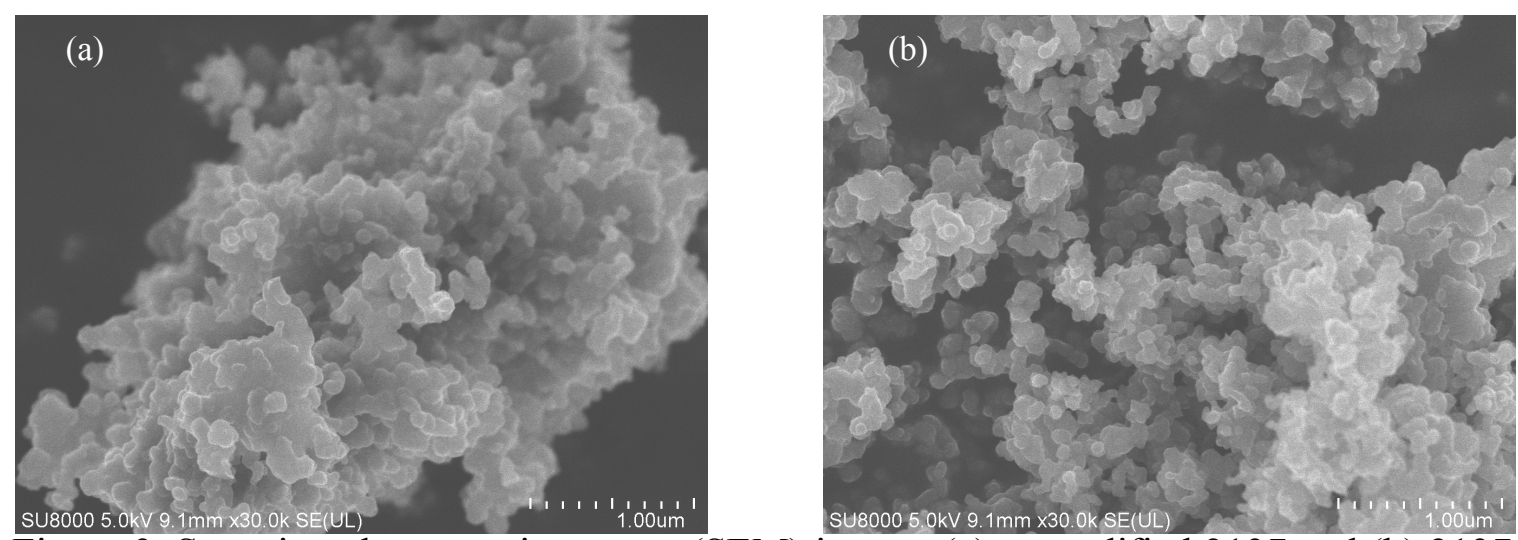

Figure 3. Scanning electron microscope (SEM) images (a) unmodified $\underline{2137}$ and (b) $\underline{2137}$ modified with $15 \mathrm{AQ}$ polycondensation polymer.

Figure 3 show SEM images of (a) unmodified 2137 and (b) 2137 modified with $15 \mathrm{AQ}$ polycondensation polymer. The modified 2137 was prepared by the reaction of $15 \mathrm{mM}$ $15 \mathrm{AQ}$ and TA with $20 \mathrm{mg}$ of 2137 in $20 \mathrm{~mL}$ of cyclohexanone at $90^{\circ} \mathrm{C}$ for 6 hours. In the image of the unmodified 2137, the structure consisted from a series of spheres with the diameter ranged from ca. 50 to $100 \mathrm{~nm}$ was observed. The structure was also observed in the image of the modified 2137. No specific difference between these two images suggests that the modification on 2137 surface is relatively uniform and the modified $15 \mathrm{AQ}$ polymer has some conductivity. 


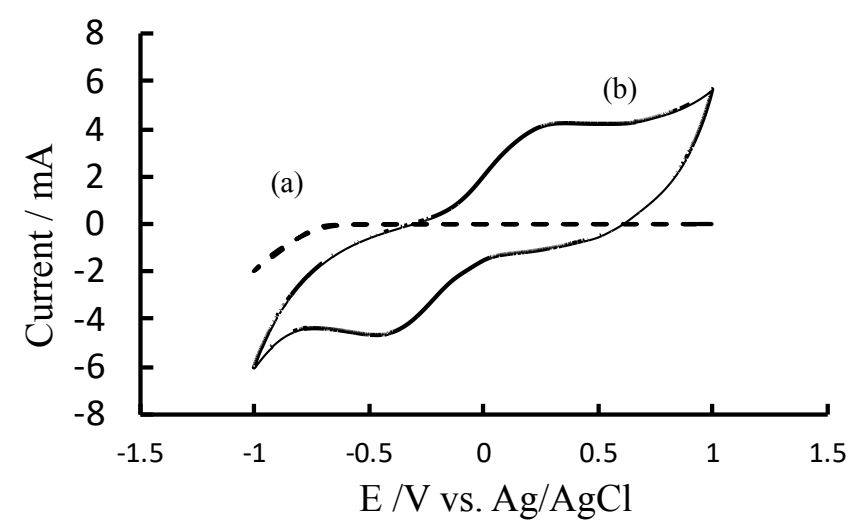

Figure 4. Cyclic voltammograms of (a) unmodified 2137 and (b) 2137 modified with the $15 \mathrm{AQ}$ polycondensation polymer in $3 \mathrm{M} \mathrm{H}_{2} \mathrm{SO}_{4}$. Scan rate: $50 \mathrm{mV} \mathrm{s}^{-1}$.

Figure 4 shows the cyclic voltammograms (CVs) of (a) unmodified $\underline{2137}$ and (b) 2137 modified with the 15AQ polycondensation polymer electrodes in $3 \mathrm{M} \mathrm{H}_{2} \mathrm{SO}_{4}$. Potential was scanned from the region between $-1.0 \mathrm{~V}$ to $1.0 \mathrm{~V}$. Both CVs were very stable for the potential cycling between this region. At the unmodified 2137 electrode the reduction current for hydrogen evolution was observed from ca. -0.8 to $-1.0 \mathrm{~V}$, however, the very small capacitive current was observed for the other potential region.

In contrast, at the modified 2137 electrode with the $15 \mathrm{AQ}$ polycondensation polymer, the large capacitive current accompanied with the redox of the anthraquinone moieties was observed. The waves at $0.3 \mathrm{~V}$ and $-0.4 \mathrm{~V}$ correspond to the oxidation and reduction of anthraquinone moieties in the 15AQ polycondensation polymer on the 2137 . This result indicates than the anthraquinone moieties in the polycondensation polymer have the redox function as shown in Figure 1(c). The capacitive and redox currents of 15AQ polymer were affected by the $\mathrm{pH}$ and concentration of the supporting electrolyte. In alkaline solution $(0.1$ $\mathrm{M} \mathrm{KOH})$, all the CB electrodes (2137, 2175, FX-35, and $\underline{\mathrm{HS}-100)}$ ) modified with the 15AQ polymer were unstable and the current was decreasing with the increase in the potential cycling between $-1.0 \mathrm{~V}$ to 1.0. The currents disappeared within ten potential cycling for all the $\mathrm{CB}$ electrodes. In this experiment the mineral oil was used as a binder for the $\mathrm{CB}$ nanoparticles. The mineral oil would not play a role in alkaline solution.

On the other hand, in $0.1 \mathrm{M} \mathrm{KCl}$ and $\mathrm{NaCl}$, the $\mathrm{CVs}$ were very stable for all the $\mathrm{CBs}$, however the observed currents were smaller than those in acidic solution. When $\mathrm{HNO}_{3}$, $\mathrm{HCl}$, and $\mathrm{H}_{2} \mathrm{SO}_{4}$ were compared as a supporting electrolyte, the largest current was obtained in the case of $\mathrm{H}_{2} \mathrm{SO}_{4}$. Also, the capacitive and redox currents also depended on the concentration of the $\mathrm{H}_{2} \mathrm{SO}_{4}$ and the best result was obtained for the $\mathrm{CV}$ in $3 \mathrm{M} \mathrm{H}_{2} \mathrm{SO}_{4}$ under our experimental conditions. Since proton transfer in involved in the redox reaction of anthraquinone, it is considered that a large current was observed because of the fast electron transfer achieved in acidic media. 


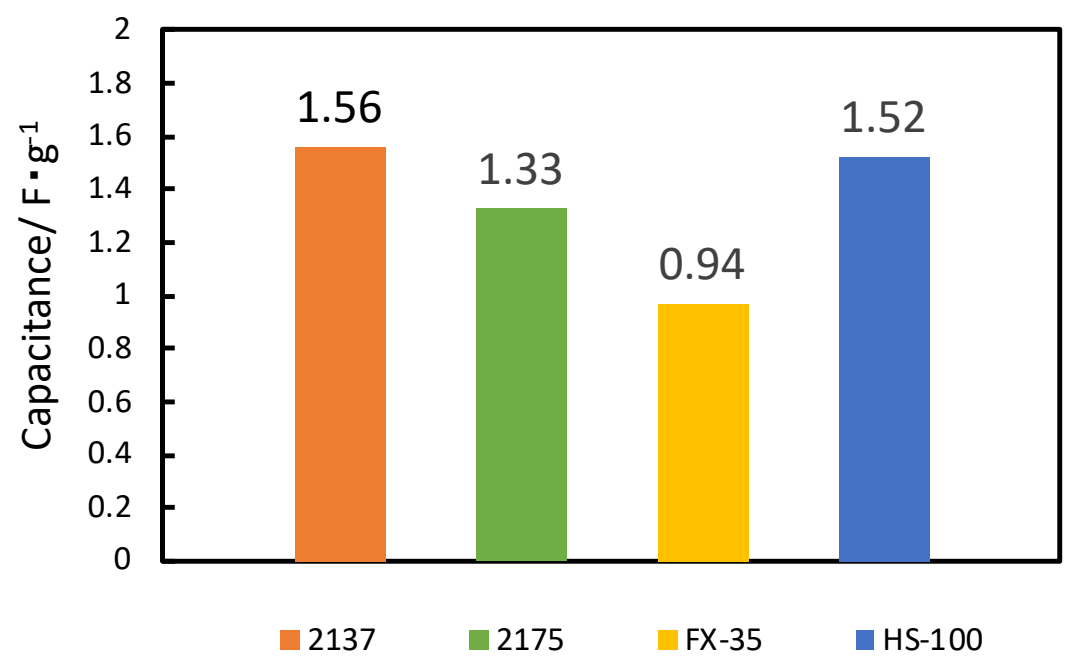

Figure 5. Effects of the kind of CBs on double layer capacitance. Data were obtained from CVs at 2137, 2175, FX-35, and HS-100 electrodes. Measurement conditions were the same as those in Fig. 4. All the CBs were modified with the polymer prepared by one-pod, one step process of $15 \mathrm{AQ}$ and $\mathrm{TA}$ under the same experimental conditions.

Figure 5 shows effects of the kind of CBs on double layer capacitance. Data were obtained from CVs at 2137, 2175, FX-35, and HS-100 electrodes. Measurement conditions were the same as those in Fig. 4. All the CBs were modified with the polymer prepared by one-pot, one step process of $15 \mathrm{AQ}$ and TA under the same experimental conditions. The capacitances shown in Figure 5 were average ones obtained from three independent experiments. Table 1 shows the average particle size and specific surface area of four kinds of CBs (2137, 2145, FX-35, and HS-100).

At first, the modification method by one-pot polycondensation polymer of $15 \mathrm{AQ}$ and TA was effective for all the CBs. All kinds of modified CBs have the functions as the EDLC. In general, the current is proportional to the surface area of the electrode. So, judging from the data in Table 1, the largest capacitance should be obtained for the FX-35 electrode having the largest specific surface area. However, the obtained result was worst among the four kinds of CBs, although the difference is not so remarkable. The largest capacitance was obtained for the 2137 electrode and the capacitance obtained at HS-100 electrode is very close to the one obtained for the 2137 electrode. In this experiment, no clear relationship between the surface area of the CBs and the capacitance was obtained. The factors other than the surface area and particle size of the nanocarbon would affect the capacitance.

TABLE I. Properties of Four kinds of Carbon Black.

\begin{tabular}{ccc}
\hline Abbreviation of Carbon Black & Average Particle Size / nm & Specific Surface Area / $\mathbf{~ m}^{\mathbf{2}} \mathbf{g}^{\mathbf{1}}$ \\
\hline$\underline{2137}$ & 35 & 69 \\
$\underline{\underline{2145}}$ & 36 & 65 \\
$\underline{\mathrm{FX}-35}$ & 23 & 133 \\
$\underline{\mathrm{HS}-100}$ & 48 & 39 \\
\hline
\end{tabular}

\section{Conclusion}


By one-pot polymerization of anthraquinone derivative having two hydroxy groups (1,5-dihydroxyanthraquinone) and aliphatic amine (1,3,5-trimethyl-1,3,5-triazinane), we succeeded in modify four kinds of carbon blacks. Although the modified CBs were unstable in the alkaline media, the electrodes show stable cyclic voltammograms in neutral and acidic media. All the modified CBs shows the function as electrochemical double layer capacitance. Especially, the largest capacitance was obtained for the $\underline{2137}$ electrode in $3 \mathrm{M}$ $\mathrm{H}_{2} \mathrm{SO}_{4}$.

\section{Acknowledgments}

The authors are grateful to Mr. Takafumi Komaki who works for Denka Co., Ltd. and provided us four kinds of Denka Blacks (carbon blacks).

\section{References}

1. P. Sae-Oui, U. Thepsuwan, and P. Thaptong, Adv. Polym. Technol., 33, 21422 (2014).

2. S. Matchawet, A. Kaesaman, P. Bomlai, and C. Nakason, Polym. Compos., 38, 1031 (2017).

3. H. Kim, M. E. Fortunato, H. Xu, J. H. Bang, and K. S. Suslick, J. Phys. Chem. C, 115, 20481 (2011).

4. L. Gan, H. Guo, z. Wang, X. Li, W. Peng, J. Wang, S. Huang, and M. Su, Electrochim, Acta, 104, 117 (2014).

5. C. Xu, L. Cheng, P. Shen, and Y. Liu, Electrochem. Commun., 9, 997 (2007).

6. T. Xia, W. Zhang, Z. Wang, Y. Zhang, X. Song, J. Murowchick, V. Battaglia, G. Liu, and X. Chen, Nano Energy, 6, 109 (2014)

7. A. Murakami, H. Noguchi, Y. Kuwahara, M. Takafuji, S. Nozato, R. Sun. A. Nakasuga, and H. Ihara, Chem. Lett., 46, 680 (2017).

8. N. Yamada, H. Noguchi, Y. Orimoto, Y. Kuwahara, M. Takafuji, S. Pathan, R. Oda, A. M. Rahimi, M. Ramazanov, and H. Ihara, Chem. Eur. J., 25, 10141 (2019).

9. K. Kinoshita and J. Bett, Carbon, 11, 403 (1973).

10. E. Gagson, J. Electrochem. Soc., 122, 521(1975).

11. S. Evans, J. Electrochem. Soc., 113, 165(1966).

12. J. Randin and E. Yeager, J. Electroanal. Chem., 36, 257(1972).

13. T. Kobayashi, H. Yoneyama, and H. Tamura, J. Electroanal. Chem., 177, 281 (1984).

14. A. Rudge, J. Davey, I. Raistric, and S. Gottesfeld, J. Power Sources, 47, 89 (1994).

15. Z. Jiang, X. Zhang, and Y. Xiang, J. Electroanal. Chem., 351, 321 (1993).

16. K. Awaya, K. Hatakeyama, M. Koinuma, and Y. Matsumoto, Chem. Lett., 46, 1030, (2017). 